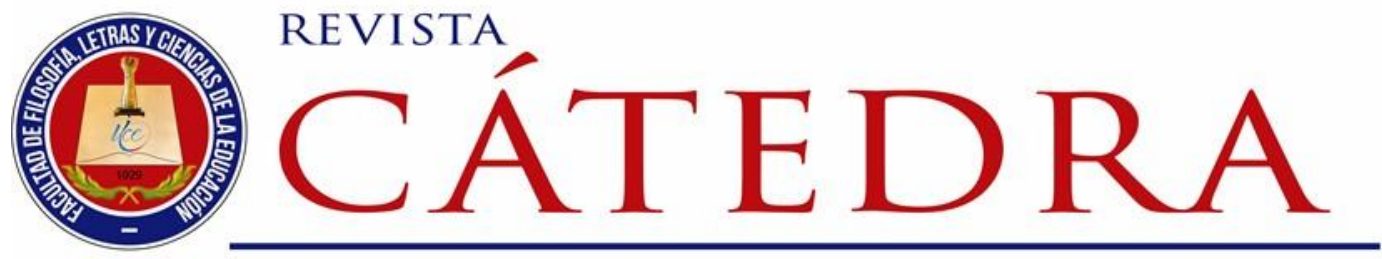

\title{
Impacto del uso de las TIC como herramientas para el aprendizaje de la matemática de los estudiantes de educación media
}

\author{
Impact of the use of ICT as tools for learning \\ mathematics for high school students \\ Jorge Revelo-Rosero \\ Universidad UTE, Quito, Ecuador \\ jorge.revelo@ute.edu.ec \\ https://orcid.org/0000-0002-2756-4856 \\ Sonia Carrillo-Puga \\ Universidad Técnica de Machala, Machala, Ecuador \\ scarrillo@utmachala.edu.ec \\ https://orcid.org/0000-0001-5026-8468
}

(Recibido: 02/08/2018; Aceptado: 16/08/2018; Versión final recibida: 02/09/2018)

Cita del artículo: Revelo-Rosero, J. y Carrillo Puga, S. E. (2018). Impacto del uso de las TIC como herramientas para el aprendizaje de la matemática de los estudiantes de educación media. Revista Cátedra, 1(1), 70-91.

\section{Resumen}

En la última década existe un gran debate sobre el impacto que tiene el uso de las Tecnologías de la Información y de la Comunicación (TIC) en el ámbito educativo. La tendencia mediática y el uso masivo de tecnologías (computadores, teléfonos inteligentes, tabletas, PDA, laptops, entre otros) con conexión a Internet, son tendencias que generan cambios en el modo de aprender y acceder al conocimiento en una sociedad digitalizada. El estudio presentado es un diseño de investigación no experimental descriptivo con un enfoque cuantitativo, con una muestra de 121 estudiantes y 29 profesores de área de matemáticas de nivel medio de las unidades educativas de las provincias de Pichincha, Guayas y el Oro; pretende aportar evidencias empíricas sobre el nivel impacto que tiene la integración de las TIC como herramientas para el aprendizaje de la matemática de los estudiantes de educación media. Los resultados muestran que el papel de la tecnología e Internet en el aprendizaje de la matemática pueden generar alguna motivación, no 
representan para los estudiantes y docentes un factor significativo ni de alto impacto en el aprendizaje de la matemática a largo plazo, no por su uso o acceso a ellas, sino por la falta de competencia para aplicarlas en su aprendizaje.

\section{Palabras clave}

Educación media, educación tecnológica, formación del profesorado, matemáticas, TIC

\section{Abstract}

In the last decade, the debate about the impact on the use of Information and communication technologies (ICT) in education has educational field has increased. The media trend and the massive use of technologies (computers, smartphones, tablets, PDAs, laptops, among others) with Internet connection are trends that generate changes for learning and accessing knowledge in a digital society. This study is a design of non- experimental descriptive research with a quantitative approach, with a student and teachers sample: 121 students and 29 teachers medium level mathematics from the educational institutions from Pichincha, Guayas and El Oro; which aims to provide empirical evidence about the level of impact of ICT integration, tools used for learning mathematics for middle school students. The results show that the role of technology and the Internet in the learning of mathematics can generate some motivation, they do not represent for the students and teachers a significant factor or high impact in the long-term learning of mathematics, not for its use or access to them, but for the lack of competence to apply them in their learning.

\section{Keywords}

High school, ICT, mathematics, teacher training, technology education

\section{Introducción}

El presente artículo tiene como objetivo determinar el nivel de impacto que tiene la integración de las Tecnologías de la Información y la Comunicación (TIC) como herramientas para el aprendizaje de la matemática de los estudiantes de educación media de Ecuador. En este sentido, es necesario considerar que toda enseñanza tiene un carácter educativo, ello supone que la educación debe brindar al ser humano no solo condiciones para su formación cognoscitiva, desarrollo de su pensamiento, de sus capacidades y habilidades, sino también para la formación de los distintos aspectos de su personalidad.

El presente siglo está caracterizado por el acelerado desarrollo científico y tecnológico; en el que, nuestra sociedad se encuentra en un profundo proceso de transformación estructural en relación con la economía global, la política, la cultura, el medio ambiente; sobre todo, con la revolución digital de las TIC cada vez más especializadas. En suma, estos hechos están relacionados con la diversidad de cosmovisiones, identidades, saberes y expresiones en un universo complejo y en permanente cambio; sobre todo, con las formas de enseñar, aprender, comunicar y trabajar.

La sociedad del conocimiento es una sociedad de personas, no de tecnologías (Castaño, 2006), se caracteriza por el constante y acelerado cambio del conocimiento asistido por el rápido progreso de las TIC que facilitan la creación, distribución y manipulación de la información, ello trae consigo que el conocimiento adquirido durante una época de formación puede quedar rápidamente obsoleto en la siguiente (Adell, 1997; Cano, 2007; 
González, 2009), donde internet juega un papel esencial en las transformaciones económicas, sociales, políticas y culturales de una sociedad (Castells, 2010). Una sociedad en la cual, las condiciones de generación, procesamiento y transmisión de la información basada en el conocimiento han sido alteradas de forma substancial por la revolución tecnológica centrada en las TIC, principalmente en las computadoras y en las redes digitales (Romeu, 2011).

En este contexto, la evolución de las TIC está impactando en el mundo de la educación. En este escenario, las instituciones educativas de todos los niveles tienen el enorme compromiso de replantearse nuevas prioridades educativas, orientando la concepción de la educación y sus enfoques pedagógicos hacia una visión integral y holística del quehacer educativo. Este proceso involucra, la formación continua y permanente del profesorado en competencias y capacidades integrales, factores claves para su integración en la enseñanza de la matemática. Por tanto, el nuevo rol del docente en general y en particular el del área de matemáticas juega un papel protagonista en la enseñanza para lograr aprendizaje significativo en sus estudiantes.

\section{Revisión de la literatura}

Hoy en día, la evolución de las TIC está impactando en el mundo de la educación, por tanto, la tecnología proporciona una amplia gama de recursos disponibles para apoyar el aprendizaje de la matemática (Revelo, Revuelta y González-Pérez, 2018). La presencia de la TIC dentro y fuera del aula ha experimentado ingentes cambios dentro del sistema educativo actual. Los retos y las exigencias a las que deben dar hoy respuesta las instituciones educativas determinan la relevancia de algunos factores, si se pretende una educación de calidad que atienda a la complejidad actual. Así, el papel a jugar por las TIC en el proceso educativo es relevante ya que aportan la posibilidad de flexibilizar y mejorar procesos que inciden directamente en el aprendizaje, la organización escolar o la comunicación con la comunidad, entre otros (González-Pérez y De Pablos, 2015). En este contexto, el proceso de implementación de las TIC al ámbito educativo, depende de los recursos tecnológicos con los que cuentan las instituciones educativas, y de las facilidades de acceso para insertarlas a la práctica pedagógica. Para ello es importante la formación permanente del profesorado sobre la importancia de dominar los medios digitales, de igual modo, en aptitudes y técnicas relacionadas para implementar prácticas pedagógicas innovadoras en el aula con TIC (Revelo, 2017).

En este contexto, Ecuador ha orientado sus políticas educativas para mejorar la calidad de la educación, mediante la promulgación de la Ley Orgánica de Educación Superior (LOES, 2010) y la Ley Orgánica de Educación Intercultural (LOEI, 2011). Dichas leyes están en concordancia con los principios constitucionales establecidos en la Carta Suprema (2008) y los instrumentos internacionales de derechos humanos que regulan los principios sobre educación en los niveles de educación inicial, básica y bachillerato. En este contexto, los nuevos desafíos del Estado ecuatoriano que busca formar profesionales y académicos con capacidades y conocimientos que respondan a las necesidades del desarrollo nacional con una visión humanista, solidaria, comprometida con los objetivos nacionales y con el buen vivir, en un marco de pluralidad y respeto.

Por otra parte, el Art. 350, de la Constitución ecuatoriana señala que:

El Sistema de Educación Superior tiene como finalidad la formación académica y profesional con visión científica y humanista: la 
investigación científica y tecnológica; la innovación, promoción, desarrollo y difusión de los saberes y las culturas: la construcción de soluciones para los problemas del país, en relación con los objetivos del régimen de desarrollo (p. 162).

En suma, que regule y garantice "el derecho a una educación superior de calidad, que propenda a la excelencia..." (LOES, 2010, p. 5). Para garantizar las condiciones de desarrollo, lograr la equidad y cobertura, es prioritario invertir en infraestructura tecnológica, equipamiento y capacitación en el uso de las TIC como herramientas de apoyo pedagógico para los docentes (Angulo et al., 2013; Braslavsky, 2000). En este sentido, la acelerada transformación que las TIC imprimen en hábitos vitales, estilos de aprendizaje y modalidades de interacción que son un mar de posibilidades tan desconocidas como infinitas a las que la educación no solo debe enfrentarse, sino también dar respuestas educativas (Pérez, Castro y Fandos, 2016).

En consecuencia, la formación permanente del docente debe ser una de las principales líneas de acción del Estado Ecuatoriano, sobre todo, ahora que el uso de las tecnologías móviles (Teléfonos inteligentes, tabletas, PDA, laptops, entre otros) por nuestros estudiantes, facilitan la implementación de metodologías más dinámicas, flexibles y abiertas para el aprendizaje de la matemática. Por tanto, el nivel de conocimiento de estos recursos tecnológicos por el profesor nos da luces acerca de su uso para generar cambios innovadores dentro de su práctica educativa para generar aprendizaje significativo en los estudiantes. Precisamente, en la actualidad con el uso de las TIC, el estudiante eleva su autonomía y responsabilidad ante su propio aprendizaje y contribuye, con su participación en el aprendizaje de sus otros compañeros de estudio cuando interactúa en entornos virtuales de aprendizaje. Es decir, que la integración de las TIC como innovación educativa aportará al desarrollo de una cultura digital mediante la creación de entornos de aprendizaje, entendiéndose éstos como "espacios organizados con el propósito de lograr el aprendizaje y que para que éste tenga lugar requiere ciertos componentes ya señalados: una función pedagógica (...), la tecnología apropiada a la misma (...) y los aspectos organizativos (...)" (Salinas, 2004, p. 2).

Es importante, destacar que la formación del profesorado debe estar asociada con la integración de las TIC al sistema educativo, puesto que, permiten nuevas posibilidades de diseño e implementación de nuevas metodologías de enseñanza - aprendizaje como parte integral del proceso educativo, que busca potenciar el aprendizaje y hacerlo más significativo en los estudiantes (Del Moral y Villalustre, 2010). En este sentido, el uso de las TIC desarrolla la competencia digital que forma parte de las competencias docentes que caracterizan el perfil profesional del profesor actual (Carrera y Coiduras, 2012). La competencia digital, al ser una de las competencias básicas del docente del siglo XXI, ha provocado que la revolución digital esté llegando a las aulas escolares a gran velocidad, introduciendo mejoras en los procesos de innovación en docencia y gestión, lo que ha propiciado un cambio en el perfil del docente y el estudiante (Cabero, 2013; Cózar y Roblizo, 2014; Esteve y Gisbert, 2012; Silva et al., 2016).

Como plantean algunas instituciones internacionales, el rol y la responsabilidad del docente es clave para que, mediante el uso de las TIC, ayudar a los estudiantes a adquirir y desarrollar las competencias necesarias para el siglo XXI (Comisión Europea, 2006, 2012 y 2013; UNESCO, 2008 y 2011). Estos deben ser capaces de apoyar el aprendizaje de sus estudiantes en un mundo digital, capaces de utilizar las TIC para mejorar y transformar las prácticas educativas dentro y fuera del aula, mediante el uso de la TIC (Hall, Atkins y Fraser,

Licencia Creative Commons Atribución 4.0 Internacional (CC BY 4.0)

Revista Cátedra, 1(1), pp. 70-91, septiembre-diciembre 2018. e-ISSN: 2631-2875

https://doi.org/10.29166/catedra.v1i1.764 
2014), lo que algunos investigadores han llamado, ser competentes para desarrollar su competencia digital como docentes.

\section{Desarrollo de la competencia matemática a través del uso de las TIC}

La rápida evolución digital de la Web 2.0 cada vez más especializada que ha transformado el Internet (Castells, 2008), como instrumento para la innovación de los procesos de enseñanza y aprendizaje, se han generado nuevos modelos de producir y compartir conocimiento e información a través de la Red (Mohammed y Ramírez, 2009; Tello Leal et al., 2010; Sangeeta, 2012). Por consiguiente, la utilización de las TIC como recurso didáctico, permite desarrollar la competencia matemática mediante la interacción en tiempo real entre estudiantes y profesores, compañeros y consigo mismo a través de la red.

Puesto que, para poder contribuir al desarrollo de la competencia digital del docente desde el área de la matemática, es necesario ir más allá de una simple definición genérica de la competencia. Para ello es preciso realizar aportaciones que tengan utilidad práctica que trasciendan a las meras aportaciones teóricas. En este contexto, en el área de matemáticas, son muchos los recursos de las TIC que han sido utilizados por el docente para insertarlos a mejorar el aprendizaje de la matemática, entre ellos se describen los blogs, wikis, foros, chats, videos, redes sociales, etc. (Basurto, 2015; López, 2011; López y Eduteka, 2003; Morón, 2013; SCOPEO, 2012).

\section{Ámbito de acción de las TIC \\ TIC / Software específico}

Gestión de la información y alfabetización informacional

Comunicación y colaboración
Navegadores Web (Mozilla, Internet Explorer, Google Chrome, etc.), Google, Google Drive, Dropbox, Wikipedia, Bing, WolframAlpha, blogs, wikis, Redes Sociales, Youtube, Symbaloo, Delicious, Diigo, Scoop.it, Storify

Foros, chats, blogs, wikis, Redes Sociales (Facebook, Twitter, Edmodo, Google+),

\section{Competencia digital docente para la enseñanza de la matemática a través del uso de las TIC}

- Uso de navegadores para buscar, localizar y filtrar información, datos y contenidos digitales sobre temas específicos del área de matemáticas.

- Organizar, evaluar y clasificar información y contenido digital disponible en la red, con fines educativos que permitan desarrollar el aprendizaje colaborativo en el área de matemáticas.

- Almacenar y recuperar información y contenido digital para mejorar el proceso de enseñanza - aprendizaje de la matemática.

- Interacción mediante la gestión, uso y aplicación de la comunicación digital. 
Colaborativas (Google Drive, Dropbox), Contenido multimedia (Youtube, Prezi, Slideshare, Scrtibd, Flickr), video conferencias, aulas virtuales,...

Blogs, Wikis, Redes Sociales

Creación y publicación de contenidos
(Facebook, Twitter, Edmodo, Google+), Colaborativas (Google Drive, Dropbox), Contenido multimedia (Youtube, Prezi, Slideshare, Scrtibd, Flickr), video conferencias, aulas virtuales,... Conexiones dinámicas manipulables como Geogebra, Cabri, Wimplot, Graph, Realidad Aumentada, WolframAlpha, Mathway, Photomath ...

Herramientas avanzadas de Excel, Cal de libre office, calculadora, Derive, Wiris, wxMaxima, SPSS, Comunidades Ricas en
- Comprender el uso adecuado de las distintas formas de comunicación a través de medios digitales.

- Compartir información y contenidos digitales a través de los distintos medios de comunicación digitales.

- Participación ciudadana en línea, mediante el uso de entornos digitales que propicien el trabajo colaborativo en el área de matemáticas.

- Desarrollar trabajo colaborativo mediante el uso canales digitales con el fin de apoyar los procesos de enseñanza - aprendizaje de la matemática.

- Utilizar y gestionar actividades de aprendizaje en comunidades virtuales y redes sociales de manera ética, legal y segura, instruyendo a mismo tiempo a sus estudiantes a tener un comportamiento responsable en la red.

Crear, rastrear y transmitir su propia identidad digital al igual que la de sus estudiantes.

- Aplicación de herramientas de la Web 2.0 para crear materiales educativos digitales (texto, presentaciones, imágenes, videos, tablas, mapas conceptuales) y los comparte en red.

- Crea y gestiona espacios de la Web 2.0 donde publica contenidos educativos multimedia (imágenes, infografías, sonidos, animaciones, vídeos...) que se adapten a las necesidades de aprendizaje de la matemática.

- Crea y gestiona contenidos específicos de matemáticas mediante el uso de: blogs, wikis, Webquest, contenidos multimedia (videos YouTube, 
Recursos Matemáticos como Proyectos Descartes, Proyecto Sócrates, Kahn Academy, Eduteka, ...
Prezi, Scribd, Slideshare,...), como innovación educativa.

- Conoce, gestiona y utiliza una amplia variedad de conexiones dinámicas manipulables, herramientas avanzadas, comunidades ricas en recursos matemáticos para adaptarlos a las necesidades de enseñanza aprendizaje de la matemática.

- Integra, combina, modifica contenido digital encontrado en la Red ajustándolo a sus necesidades y respetando licencias de uso.

- Respeta la normativa legal sobre derechos de autor de los contenidos digitales de la red, citando sus fuentes.

- Realiza modificaciones en programas informáticos, aplicaciones, configuraciones, programas, dispositivos para usarlos como innovación educativa.

- Realiza modificaciones a las funciones avanzadas de medios digitales en relación con las necesidades de su tarea docente.

- Realiza modificaciones a software libre con la finalidad de mejorarlo y adaptarlo a las necesidades del proceso enseñanza-aprendizaje de la matemática.

\subsection{Ventajas e inconvenientes del uso de las TIC en el aprendizaje de la matemática} Las TIC permiten al estudiante ir construyendo un puente entre las ideas intuitivas y los conceptos matemáticos formales, proporcionando un ambiente adecuado mediante la interacción, visualización, interactividad; facilitando por tanto, el aprendizaje por descubrimiento de los estudiantes (López, 2010). En este contexto, las TIC como recurso didáctico están promoviendo una nueva visión del conocimiento y del aprendizaje (Freire, 2007), transformado el rol del docente dentro del proceso de enseñanza - aprendizaje, insertándolo en la dinámica de creación y diseminación del conocimiento y a través de la Red.

Alonso et al. (2010), Area-Moreira (2010), Area-Moreira y Ribeiro-Pessoa (2012), Bennett et al (2012), Buckingham (2009), Cela et al (2010), Colás y Casanova (2010), Cobo y Pardo (2007), Del Moral y Villalustre (2010), De la Torre (2006), Freire (2007), Kopcha (2012), 
Molina e Iglesias (2014), Pachler et al (2010), Revelo (2017), Revuelta y Pérez (2009), Romero (2008), Salinas, Benito y Lizana (2014), Santamaría (2005), Wong et al (2008), Yang (2012), Zuluaga, Pérez y Gómez (2012), y otros autores, llegan a la conclusión que la integración de las TIC en la enseñanza de la matemática aporta múltiples ventajas en el mejoramiento de la calidad docente, materializadas en aspectos tales como el acceso desde áreas remotas, la flexibilidad en tiempo y espacio para el desarrollo de las actividades de aprendizaje (Ferro, Martínez y Otero, 2009). Las TIC permiten además buscar, interactuar, recopilar y procesar información para generar nuevos conocimientos.

\section{TIC en el aprendizaje de la matemática}

Tipos

Aplicaciones

\section{Ventajas}

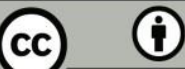

Revista Cátedra, 1(1), pp. 70-91, septiembre-diciembre 2018. e-ISSN: 2631-2875
- Blogs

- Wikis

- Redes sociales

- De colaboración

- Marcadores sociales

- Contenidos multimedia

- Otras herramientas

- Fomentar y desarrollar la expresión escrita.

- Fomentar y desarrollar espacios de discusión e intercambio de información.

- Reforzar las actividades de aula y desarrollo de ejercicios sobre temas tratados en la misma.

- Crear, buscar, editar, gestionar y publicar contenidos e información con fines educativos.

- Motivar la participación interactiva entre docentes y estudiantes.

- Establecer espacios de integración de todo tipo de enlaces, texto, imágenes, audio, vídeo, presentaciones o animaciones y cualquier otro elemento multimedia.

- Fomentar el trabajo individual, colaborativo y corporativo los participantes.

- Permitir la construcción y desarrollo del conocimiento dentro de una comunidad de aprendizaje.

- Fomentar y desarrollar la formación del profesorado.

- Fomentar y desarrollar Competencias Digitales tanto en docentes como estudiantes.

- Facilitan el acceso inmediato a la búsqueda y selección dela información disponible en la Red desde cualquier lugar.

- Permiten configurar contenido hipertextual y multimedia sobre cualquier temática, como es el caso de la matemática.

- Permiten crear, editar, gestionar, publicar y compartir por los diferentes canales de comunicación digital (foros, chats, blogs, wikis, redes sociales, entre otros) contenidos e información con fines educativos.

- Facilitan las relaciones con redes sociales y otras aplicaciones de la red.

- Establecen ruptura de las barreras espacio - temporales enlas actividades de enseñanza - aprendizaje.

- No requiere de grandes conocimientos informáticos, con un nivel 
Inconvenientes de usuario cualquiera puede usar las herramientas Web 2.0

- Facilitan la comunicación e interacción entre los distintos agentes del proceso enseñanza - aprendizaje ya síncrona y asincrónicamente.

- Favorecen el trabajo individual, colaborativo y cooperativo delos participantes.

- Propician distintos niveles de participación individual o colectiva entre docentes y estudiantes.

- Permiten un alto grado de interdisciplinaridad para la educación ya que permiten romper esquemas tradicionales de enseñanza aprendizaje dentro y fuera del aula universitaria.

- Son dinámicas, fomentan procesos formativos abiertos y flexibles para el aprendizaje autónomo y colaborativo de los estudiantes desde cualquier lugar.

- Permiten a los estudiantes reflexionar sobre su proceso de aprendizaje.

- Permiten el aprendizaje a partir de los errores (Feedback)

- Aumentan el interés y la motivación de los estudiantes con dificultades para mejorar su proceso de aprendizaje.

- Facilitan la construcción del conocimiento dentro de una comunidad de aprendizaje.

- Fomentan el desarrollo y formación del profesorado.

- Permiten adquirir competencias digitales para ser funcional en la sociedad de la información y el conocimiento.

- Favorecen la participación docentes y estudiantes en comunidades virtuales y redes sociales, herramientas sociales y colaborativas para promover la reflexión, creación, empoderamiento y auto-desarrollo.

- Propagación inmediata de contenidos e información (RSS) que permitan un mejor desarrollo de la estructura de la Red.

- Confidencialidad de la información publicada en la Red.

- La información y el contenido es público en la Red.

- Mucho contenido sin fundamentación científica o fuentes de credibilidad.

- Inseguridad del almacenamiento de datos en la Red.

- Dependencia completa del acceso a Internet.

- Cambios en las condiciones del servicio: pueden ser gratuitas hoy y mañana no.

- Vulnerabilidad de la propiedad intelectual - Derechos de autor sobre el contenido e información digital que se publica en la Red.

- Desconocimiento y temor al uso y aplicaciones de la Web 2.0 en procesos educativos por los participantes.

- La enseñanza es no personalizada.

- Exceso de información, la cual es difícil de procesar ensu totalidad 


\section{Objetivos de la investigación}

Considerando el contexto donde tiene lugar la investigación que son instituciones de educación media de Ecuador, los objetivos a dar respuesta son:

1. Determinar el nivel formación que tienen docentes y estudiantes en el uso de las TIC como herramientas para el aprendizaje de la matemática.

2. Analizar el nivel de impacto que tiene integración de las TIC como herramientas para el aprendizaje de la matemática de los estudiantes de educación media.

Para dar respuesta a los objetivos propuestos se plantearon los siguientes interrogantes de investigación:

1. ¿El nivel de formación que tienen docentes y estudiantes en el uso de las TIC influye en el aprendizaje de la matemática?

2. ¿Tiene impacto la integración de las TIC en el aprendizaje de la matemática de los estudiantes de educación media?

\section{Métodos y materiales}

Se abordó un diseño de investigación no experimental descriptivo con un enfoque cuantitativo que al ser un proceso formal, objetivo y sistemático permitió obtener información cuantificable sobre un fenómeno investigado en forma numérica, mediante el empleo de pruebas estadísticas se pudo describir, explicar y probar las interrogantes planteadas en la misma (Bisquerra, 2004). Para la recogida de los datos se diseñó como único instrumento de recolección de información una encuesta estructurada con el fin de obtener la información referente a los objetivos planteados. La encuesta tuvo dos tipos de preguntas cerradas donde se le daba la opción a los encuestados de elegir si, no y otras de opción múltiple. El cuestionario fue aplicado de manera física por estudiantes de las Carreras de Educación del Sistema de Educación a Distancia de la Universidad UTE y fue la misma para estudiantes y profesores de unidades educativas de las provincias de Pichincha, Guayas y el Oro.

\section{Población y muestra}

La población con la cual se trabajó fue de 458, de los cuales 423 son estudiantes de educación media y 35 docentes del área de matemáticas correspondientes a unidades educativas de las provincias de Pichincha, Guayas y el Oro. Para el cálculo de la muestra de la población de 458 estudiantes y docentes, según el caso se utilizó la fórmula estadística para tamaños de población mayor a 10 personas con la cual se obtuvieron 150 unidades muéstrales. Distribuidas de la siguiente manera: 29 docentes del área de matemáticas y 121 estudiantes, a los cuales se les solicitó responder voluntaria y anónimamente la encuesta presentada de manera física con el fin de garantizar los resultados y conclusiones generadas de la información recolegida en la presente investigación (Ver cuadro 3). 


\begin{tabular}{lcccc}
\multirow{2}{*}{ Provincia } & \multicolumn{2}{c}{ Población } & \multicolumn{2}{c}{ Muestra } \\
& Estudiantes & Profesores & Estudiantes & Profesores \\
Guayas & 116 & 23 & 40 & 17 \\
Pichincha & 240 & 8 & 49 & 8 \\
El Oro & 67 & 4 & 32 & 4 \\
Total & 423 & 35 & 121 & 29
\end{tabular}

Cuadro 3. Estrategia probabilística para seleccionar la muestra

\section{Planificación y aplicación del trabajo de campo}

Etapa I: En esta etapa se determina la presentación del proyecto de investigación dividido en tres planes de titulación. Una vez aprobados los mismos se procede búsqueda y revisión de la literatura específica referente al tema de investigación en algunas bases de datos como Education Resource Information Center (ERIC), Scientific Electronic Library Online (SciELO), Dialnet, Google Académico, Tesis en Red (TDR), Tesis Doctorales en Línea (TDX), Tesis Doctorales desde 1976 (TESEO), Scopus (Multiplidisciplinar), Host Research Databases (EBSCO), Biblioteca Digital de la OEI, Biblioteca de la UEX, Eduteka, revistas especializadas, entre otras. Esta etapa de investigación se empezó a desarrolla a partir de abril de 2016.

Etapa II: está determinada por la definición del diseño de la investigación de tipo no experimental de corte cualitativa, enfocado en un sondeo de tipo descriptivo y la definición de la encuesta como técnica para la recogida de la información que busquen dar respuesta a los objetivos planteados.

Etapa III: Se diseña la encuesta, se define la población y muestra. La técnica aplicada para la elección de la muestra es de tipo probabilístico, por tanto, para calcular la muestra representativa de la población se utilizó la fórmula estadística. "Las muestras probabilísticas son esenciales en los diseños de investigación transversal, tanto descriptivos como correlacionales-causales" (Hernández Sampieri et al., 2010, p. 177). Dadas las características de la investigación, todos los elementos de la población tienen una misma probabilidad de ser elegidos. Una vez, establecido el cuestionario se procede a su aplicación de manera física por estudiantes de las Carreras de Educación del Sistema de Educación a Distancia de la Universidad UTE y fue la misma para estudiantes y profesores de unidades educativas de las provincias de Pichincha, Guayas y el Oro, previa autorización de las autoridades académicas y aprobación de los estudiantes y profesores seleccionados al azar para su posterior análisis e interpretación. El tiempo aproximado para contestar el cuestionario fue de 5 a 10 minutos, y la aplicación fue el mes de octubre de 2016 .

Etapa IV: Se realiza el análisis estadístico e interpretación de los datos recogidos a través del instrumento elaborado para la presente investigación se empleó el método cuantitativo descriptivo de frecuencias y porcentajes de cada una de las variables del presente estudio. El análisis se complementó por medio de distribuciones de frecuencia de tipo bivariado mediante tablas de contingencia, el empleo de las pruebas estadísticas de chi-cuadrado y del coeficiente de contingencia y un nivel de confianza del 95\% con el fin de determinar la relación estadística entre las variables cruzadas. Se empleó el programa estadístico SPSS para Windows, versión 22.0. Finalmente, se procede a la redacción y presentación de los resultados junto a la discusión y conclusiones de la presente investigación. 


\section{Resultados}

Para dar respuesta a la primera pregunta de investigación ¿El nivel de formación que tiene el docente en el uso de las TIC influye en la enseñanza de la matemática?

La muestra de estudio son docentes del área de matemáticas y estudiantes del nivel medio de unidades educativas de Ecuador, de los cuales el 38,0\% $(n=57)$ corresponde a la provincia de Guayas, en igual porcentaje a Pichincha y el Oro con el 24,0\% $(n=36)$. El perfil general de los encuestados muestra una población de género femenino es casi el doble de la masculina con un $62,7 \%(n=94)$, de los cuales $81,9 \%$ son estudiantes y el $18,1 \%$ son profesoras frente a un $37,3 \%(n=56)$ son de género masculino, de los cuales $78,6 \%$ son estudiantes y el $21,4 \%$ son profesores. En cuanto al nivel de formación académica del profesorado ( $n=29)$, se puede observar que un 17,2\% ( $n=5)$ tienen estudios de Maestría, en igual porcentaje son normalistas; $55,2 \%(n=16)$ tienen título de licenciado y un $10,3 \%(n=3)$ son bachilleres, lo cual evidencia que el mayor número de profesores son profesionales en educación. En suma, se puede observar que el profesorado del área de matemáticas tiene un interés por mantenerse actualizado.

En lo que respecta a la edad se puede resaltar que la mayoría de la población estudiada pertenece a la "Generación Net" (Tapscott, 2010) o nativos digitales (Prensky, 2001), términos se utilizan para etiquetar la generación nacida después de 1980, aquellas personas cuyas "preferencias en el aprendizaje tienden hacia el trabajo en equipo, las actividades experienciales, y el uso de tecnología" (Cabra y Marciales, 2009). En el cuadro 4 , se puede observar que un 93,3\% $(n=140)$ de la muestra analizada en esta investigación es menor a 40 años, frente a un 6,7\% $(n=10)$ es mayor a 40 años, lo cual evidencia que el mayor número de estudiantes y profesores son nativos digitales y hay un número no muy significativo que si podría ser considerados como no nativos digitales y, por tanto, en cierta forma la integración de las TIC al aprendizaje de la matemática es un gran desafío (Cabra y Marciales, 2009). Investigadores como Wodzicki et al. (2012), Bennett et al. (2008) y De la Hoz, Acevedo y Torres (2015), infieren que los nativos digitales han desarrollado habilidades y destrezas que van de la mano con la evolución de la tecnología y de Internet. Esto hace que existan diferencias entre otras generaciones anteriores en el sentido de afrontar otras dificultades a la hora de implementar cambios en sus formas de aprender con el uso de las TIC.

\begin{tabular}{lcccc}
\multicolumn{1}{c}{ Edad } & \multicolumn{2}{c}{ Estudiantes } & \multicolumn{2}{c}{ Docentes } \\
& $\mathrm{n}$ & $\%$ & $\mathrm{n}$ & $\%$ \\
$10-15$ años & 50 & $33,3 \%$ & 0 & $0,0 \%$ \\
$16-20$ años & 65 & $43,3 \%$ & 0 & $0,0 \%$ \\
$21-25$ años & 5 & $3,3 \%$ & 2 & $1,3 \%$ \\
$26-30$ años & 0 & $0,0 \%$ & 5 & $3,3 \%$ \\
$31-35$ años & 0 & $0,0 \%$ & 5 & $3,3 \%$ \\
36- 40 años & 0 & $0,0 \%$ & 8 & $5,3 \%$ \\
más de 41 años & 0 & $0,0 \%$ & 10 & $6,7 \%$ \\
Total Cuadro 4. Rango de edades de la muestra estudiada $(\mathrm{n}=150)$
\end{tabular}

Licencia Creative Commons Atribución 4.0 Internacional (CC BY 4.0) 
Para el tratamiento y análisis de la información cuantitativa, se utilizó el programa estadístico SPSS 22. Para determinar que el cuestionario tenga validez y fiabilidad de constructo, se utilizó la técnica de análisis factorial y alfa de Cronbach respectivamente. Dentro del cuestionario, se agruparon los ítems por dimensiones, y una vez depurado estadísticamente el cuestionario, el factor que incluye los ítems relacionados con el nivel impacto que tiene la integración de las TIC como herramientas para el aprendizaje de la matemática de los estudiantes de educación media mostrando un índice KMO de 0,701 (> 0,5) y un Alpha de Cronbach de 0,694 (Alpha Std. = 0,679), valores aceptables para este tipo de análisis. En el cuestionario se les pidió a los docentes y estudiantes encuestados que valoren en una escala tipo Likert de 1 a 5 , donde 1 es la menor puntuación y 5 la máxima los ítems que conforman la misma.

El Cuadro 5 recoge las respuestas sobre los ítems de la dimensión Nivel de formación en el uso de las TIC como herramientas para el aprendizaje de la matemática en función de su valoración media y desviación estándar. Los resultados muestran que el factor más relevante tanto para docentes como estudiantes es el de que "consideran necesario recibir formación permanente sobre el uso y aplicación de las TIC como herramientas para desarrollar innovaciones educativas y buenas prácticas educativas" (media $=3,39$ ). Por el contrario, el factor con menor impacto es el relacionado con el que "el nivel de formación recibida en su institución educativa sobre el uso de TIC para innovar su práctica educativa en el aprendizaje de la matemática" es muy poco o escaso (media $=1,54$ ). Es decir, que en el $85,7 \%$ de los ítems están por debajo de una media del 3,0, lo que muestra una clara tendencia negativa en el nivel de formación en el uso de las TIC como herramientas para el aprendizaje de la matemática. La dispersión de las puntuaciones de cada ítem no es muy grande con respecto a la media (media global $=2,67$ ), debido a que la desviación estándar se encuentra en una escala menor a 1.

II. Nivel de formación en el uso de las TIC como herramientas para el aprendizaje de la matemática

1 Valore el nivel de conocimiento que tiene en cuanto al uso de las TIC para el aprendizaje de la matemática.

2 Conoce los recursos tecnológicos que existen en su institución para el desarrollo de su práctica educativa Considera que el uso de las TIC en el aula de clase

3 genera cambios e innovaciones en el aprendizaje de la matemática.

Usa las herramientas tecnológicas disponibles en su institución educativa tales como computadores,

4 dispositivos móviles, software de matemáticas,
internet, etc., el del proceso de enseñanza y aprendizaje.

Existe el suficiente apoyo de su institución educativa

5 para incorporar las herramientas tecnológicas como innovación educativa.

$2,43 \quad 0,789$

Valore el nivel de formación recibida en su institución

6 educativa sobre el uso de TIC para innovar su práctica educativa en el aprendizaje de la matemática

7 Considera necesario recibir formación permanente sobre el uso y aplicación de las TIC como

$\begin{array}{lll}\text { Media } & \begin{array}{c}\text { Desv. } \\ \text { Estándar }\end{array} & \mathrm{N} \\ 2,67 & 0,690 & 150 \\ 2,77 & 0,670 & 150 \\ 2,97 & 0,634 & 150 \\ & & \\ 2,91 & 0,780 & 150 \\ & & \\ 2,43 & 0,789 & 150 \\ & & 150 \\ 1,54 & 0,672 & 150 \\ & & \end{array}$

Licencia Creative Commons Atribución 4.0 Internacional (CC BY 4.0)

Revista Cátedra, 1(1), pp. 70-91, septiembre-diciembre 2018. e-ISSN: 2631-2875

https://doi.org/10.29166/catedra.v1i1.764 
herramientas para desarrollar innovaciones educativas y buenas prácticas docentes.

Total de la media global

\begin{abstract}
* 1 = Muy poco; 2 = Poco; 3 = Suficiente; 4 = Bastante; 5 = Mucho
Cuadro 5. Nivel de formación en el uso de las TIC como herramientas para el aprendizaje de la matemática $(n=150)$
\end{abstract}

Finalmente, para dar respuesta a la segunda pregunta de la presente investigación ¿tiene impacto la integración de las TIC en el aprendizaje de la matemática de los estudiantes de educación media?

El Cuadro 6 resume los ítems relacionados con el impacto de la integración de las TIC como herramientas para el aprendizaje de la matemática, en función de su valoración media y desviación estándar. Los resultados de la muestra encuestada determinan que el ítem (10) es el factor más relevante, ya que tanto docentes como estudiantes de nivel medio consideran que la integración de las TIC tendrá bastante impacto en el aprendizaje de la matemática (media $=3,59$ ), lo cual puede tener un efecto contraproducente en sus creencias de que el aprendizaje de la matemática es más eficiente si se usa TIC. Del mismo modo, los ítems (17), (11) y (15) referidos a la motivación para el tiempo de uso de recursos tecnológicos (computador, tablets, software de matemáticas, internet, etc.) serían suficientes para el aprendizaje de la matemática; aunque, las medias estén por encima de 3,0 no se aprecia con claridad en la muestra estudiada que el uso de recursos tecnológicos tengan el impacto esperado para el aprendizaje de la matemática. Por otra parte, el ítem (8) cuya media $=2,39$ (como el resto de los ítems que se encuentran por debajo de la media 3,0) se refiere a la disponibilidad recursos tecnológicos para el desarrollo de la práctica educativa en cada una de las instituciones de la muestra estudiada es incipiente o poca, lo que muestra que es un factor muy negativo a la hora de integrar las TIC para el aprendizaje de la matemática en las instituciones educativas de nivel medio estudiadas. Por otra parte, la dispersión de las puntuaciones de cada ítem no es muy grande con respecto a la media (media global $=2,91$ ), debido a que la desviación estándar se encuentra en una escala menor a 1.

III. Nivel de impacto sobre la integración de TIC en el aprendizaje de la matemática

Media $\begin{gathered}\text { Desv. } \\ \text { estándar }\end{gathered}$

En su institución existe disponibilidad recursos

8 tecnológicos para el desarrollo de su práctica educativa

9 Existe disponibilidad de conectividad a internet que facilite su labor educativa dentro del aula. Considera que el uso de las TIC mejoraría el aprendizaje de la matemática

$\begin{array}{lll}2,39 & 0,722 & 150 \\ 2,71 & 0,994 & 150 \\ 3,59 & 0,991 & 150\end{array}$

Le gustaría usar recursos tecnológicos como

11 computador, tablets, software de matemáticas, internet, etc., para mejorar el aprendizaje de la matemática

Si en las clases de matemáticas se usaran recursos

12 tecnológicos como computador, dispositivos móviles, software de matemáticas, internet, etc., el aprendizaje sería más motivador. 
El uso de redes sociales, blogs, wikis, contenidos

multimedia (videos YouTube, Prezi, Scribd,

Slideshare, ...), contribuirían a un aprendizaje

significativo de la matemática

El uso redes sociales (facebook, twitter, google plus,

etc.), blogs o páginas diseñadas por los docentes

apoyaría el aprendizaje de matemáticas por fuera del

$\begin{array}{lll}2,75 & 0,926 & 150 \\ 3,16 & 0,997 & 150 \\ 2,58 & 0,869 & 150 \\ 3,38 & 0,981 & 150 \\ 2,66 & 0,961 & 150\end{array}$
aula de clase.

15 Si pudiera usar más tiempo el computador mi aprendizaje de matemáticas sería más fácil.

El uso de softwares de matemáticas facilita el

16 aprendizaje de matemáticas más que estudiando en libros.

17 El uso de computador e Internet me ayuda a aprender

fácilmente el conocimiento de la matemática

2,91

18 El uso del computador me puede dismin Media global

* 1 = Muy poco; 2 = Poco; 3 = Suficiente; 4 = Bastante; 5 = Mucho

Cuadro 6. Impacto de la integración de TIC como herramientas para el aprendizaje de la matemática $(\mathrm{n}=150)$

\section{Discusión y conclusiones}

Los resultados presentados en este trabajo son parte de una investigación más profunda. Estos indican que la mayoría de docentes y estudiantes tienen opiniones negativas acerca del conocimiento que tienen sobre el uso de las TIC como herramientas que pueden aportar al aprendizaje de la matemática. En ello, incide de manera importante la necesidad de formación sobre el uso y aplicación de las TIC como herramientas didácticas que permitan elevar la calidad de la educación, generar mayor comunicación e interacción entre docentes y estudiantes propiciando el aprendizaje colaborativo. El nivel de formación en el uso de las TIC como herramientas para el aprendizaje de la matemática presenta una posibilidad importante en la redefinición de la práctica pedagógica en la educación. Sin embargo, las TIC al tener potencialidades pedagógicas de aplicación, implican nuevos retos para el docente que tiene bajo su responsabilidad a estudiantes que han desarrollado habilidades y destrezas que van de la mano con la evolución de la tecnología y deInternet.

Por otra parte, la incorporación de las TIC al proceso educativo significa adaptación e innovación, puesto que, el desarrollo y evolución de la tecnología es pieza clave en la sociedad actual, aunque, no es la solución mágica a los problemas educativos. Es decir, las TIC no pueden cambiar por sí mismas los procesos de enseñanza - aprendizaje, pero, si pueden aumentar ilimitadamente sus efectos en el proceso educativo. En este sentido, varios investigadores como Molina \& Iglesias (2014), Salinas, Benito, \& Lizana (2014), Padilla, Moreno, \& Hernández (2015), Rodríguez (2010), Rodríguez (2015), Sosa (2015), Revelo (2017), entre otros, en sus estudios afirman que la simple incorporación de las TIC no garantiza, en sí mismas, la transformación de las prácticas educativas. Es decir, que éstas por si solas no que modifican los procesos de enseñanza - aprendizaje, sino la manera como el profesorado las utilizan en cada área del conocimiento para que sus estudiantes mejoren su aprendizaje. 
Aunque, los resultados de la presente investigación no son concluyentes, es claro que la integración las TIC como herramientas para el aprendizaje de la matemática de los estudiantes va más allá del simple uso de estas nuevas herramientas, lo que traerán consigo cambios sustanciales en el proceso de enseñanza - aprendizaje, en la desempeño del docente, en los métodos de enseñanza, etc. En conclusión, es necesario desarrollar una mejor comprensión del uso de las TIC, de modo que, las innovaciones en el campo educativo no sean absorbidas por la tecnología, sino que, estén orientadas por los procesos pedagógicos que generen aprendizaje significativo entre docentes y estudiantes.

\section{Bibliografía}

Alonso, C., Casablancas, S., Domingo, L., Guitert, M., Moltó, O., Sánchez, J. A. y Sancho, J. M. (2010). De las propuestas de la Administración a las prácticas del aula. Revista de Educación, 352, 53-76.

Angulo Armenta, J., Mortis Lozoya, S. V., Pizá Gutiérrez, R. I. y García López, R. I. (2013). Estudio sobre competencias digitales en profesores de secundaria.

Area-Moreira, M. (2010). El proceso de integración y uso pedagógico de las TIC en los centros educativos. Un estudio de casos. Revista de Educación, 352, 77-97.

Area-Moreira, M. y Ribeiro-Pessoa, M. T. (2012). From Solid to Liquid: New Literacies to the Cultural Changes of Web 2.0. Comunicar, 19(38), 13-20. Recuperado de https://www.revistacomunicar.com/pdf/preprint/38/01-PRE-12378.pdf

Basurto Hidalgo, E. (2015). Creando certeza en las ideas matemáticas vía el uso de tecnología digital. Cuadernos de Investigación y Formación en Educación Matemática, (15), 349-360. Recuperado de http://revistas.ucr.ac.cr/index.php/cifem/article/download/23842/24010

Bennett, S., Bishop, A., Dalgarno, B., Waycott, J. y Kennedy, G. (2012). Implementing Web 2.0 technologies in higher education: A collective case study. Computers \& Education, 59(2), 524-534. https://doi.org/10.1016/i.compedu.2011.12.022

Bennett, S., Maton, K. y Kervin, L. (2008). The "digital natives" debate: A critical review of the evidence. British Journal of Educational Technology, 39(5), 775-786.

Bisquerra Alzina, R. (Coord). (2004). Metodología de la investigación educativa. La Muralla. Recuperado de https://dialnet.unirioja.es/servlet/libro?codigo=5826

Braslavsky, C. (2000). La educación secundaria en América Latina, Prioridad de la Agenda 2000. Dakar. Recuperado de http://info.worldbank.org/etools/docs/library/109259/la\%20ed\%20Sec\%20en\% 20AL\%20C\%20Braslavsky.pdf

Buckingham, D. (2009). The future of media literacy in the digital age: some challenges for policy and practices. En Veniers, P. (Ed.). Euromeduc. Media Literacy in Europe. Controversies, Challenges and Perspectives (pp. 13-24). Bruxelles: Média Animation.

Cabero Almenara, J. (2013). El aprendizaje autorregulado como marco teórico para la aplicación educativa de las comunidades virtuales y los entornos personales de aprendizaje. Teoría de la Educación; Educación y Cultura en la Sociedad de la Información, 14(2), 133. 
Cabra Torres, F. y Marciales Vivas, G. P. (2009). Mitos, realidades y preguntas de investigación sobre los 'nativos digitales': una revisión. Universitas Psychologica, 8(2), 323-338.

Cano García, E. (2007). Las competencias de los docentes. En Lola Abelló Planas, El desarrollo de competencias docentes en la formación del profesorado. Madrid: Ministerio de Educación.

Carrera Farrán, F. X., \& Coiduras Rodríguez, J. L. (2012). Identificación de la competencia digital del profesor universitario: un estudio exploratorio en el ámbito de las Ciencias Sociales. Identifying the digital competence of university lecturers: an exploratory study in the field of Social Science. Revista de docencia universitaria, 10(2), 273-298.

Recuperado de http://0search.ebscohost.com.lope.unex.es/login.aspx?direct=true \&db=a9h\&AN=90565438 \&lang=es\&site=eds-live

Castaño Collado, C. (2006). Alfabetización digital, inclusión y género. En Casado Ortiz, $R$. (Ed.). Claves de la alfabetización digital (pp. 67-76). Fundación Telefónica. Recuperado de https://dialnet.unirioja.es/servlet/articulo?codigo=3267965

Castells, M. (2008). Creatividad, innovación y cultura digital. Un mapa de sus interacciones. Telos. Cuadernos de comunicación e innovación, 77. Recuperado de https://telos.fundaciontelefonica.com/telos/articulocuaderno.asp@idarticulo=2\&re $\underline{\mathrm{v}=77 . \mathrm{htm}}$

Castells, M. (2010). La dimensión cultural del Internet. Cultura y Sociedad del Conocimiento: Presente y Perspectivas de Futuro. Recuperado de http://www.uoc.edu/culturaxxi/esp/articles/castells0502/castells0502.html

Cela Rosero, K., Fuentes, W., Alonso, C. y Sánchez, F. (2010). Evaluación de herramientas web 2.0, estilos de aprendizaje y su aplicación en el ámbito educativo. Journal of Learning Styles, $3(5)$. Recuperado de http://learningstyles.uvu.edu/index.php/ils/article/view/123

Cobo Romaní, C. y Pardo Kuklinski, H. (2007). Planeta Web 2.0. Inteligencia colectiva o medios fast food. España: Grup de Recerca d'Interaccions Digitals. Recuperado de http://site.ebrary.com/lib/alltitles/docDetail.action?docID=10378388

Colás Bravo, P. y Casanova Correa, J. (2010). Variables docentes y de centro que generan buenas prácticas con TIC. Education in the Knowledge Society (EKS), 11(1), 121-147. Recuperado de http://revistas.usal.es/index.php/revistatesi/article/view/5791

Cózar Gutiérrez, R. y Roblizo Colmenero, M. J. (2014). La competencia digital en la formación de los futuros maestros: percepciones de los alumnos de los Grados de Maestro de la Facultad de Educación de Albacete. RELATEC. Recuperado de http://dehesa.unex.es:8080/xmlui/handle/10662/2940

De la Hoz, L. P., Acevedo, D. y Torres, J. (2015). Uso de redes sociales en el proceso de enseñanza y aprendizaje por los estudiantes y profesores de la Universidad Antonio Nariño, Sede Cartagena. Formación universitaria, 8(4), 77-84.

De la Torre, A. (2006). Web Educativa 2.0. Edutec. Revista Electrónica de Tecnología Educativa, O(20). Recuperado de http://www.edutec.es/revista/index.php/edutece/article/view/515 
Del Moral Pérez, M. E. y Villalustre Martínez, L. (2010). Formación del profesor 2.0: desarrollo de competencias tecnológicas para la escuela 2.0. MAGISTER: Revista miscelánea de investigación, 23, 59-69. Recuperado de http://dialnet.unirioja.es/descarga/articulo/3403432.pdf

Ecuador. Asamblea Constituyente (2008). Constitución de la República del Ecuador. Recuperado de http://repositorio.dpe.gob.ec/bitstream/39000/638/1/NN-001Constituci\%C3\%B3n.pdf

Ecuador. Presidencia de la República. (2010). Ley Orgánica de Educación Superior. Quito: Registro Oficial Suplemento No. 298. Recuperado de http://www.ces.gob.ec/index.php?option=com phocadownload\&view=file\&id=651 $\underline{\text { \&Itemid }=564}$

Ecuador. Presidencia de la República. (2011). Ley Orgánica de Educación Intercultural. Quito: Segundo Suplemento - Registro Oficial No. 147. Recuperado de http://www.wipo.int/edocs/lexdocs/laws/es/ec/ec023es.pdf

Esteve Mon, F. y Gisbert Cervera, M. (2012). La competencia digital de los estudiantes universitarios: Definición conceptual y análisis de cinco instrumentos para su evaluación. Recuperado https://www.researchgate.net/profile/Francesc Esteve/publication/233721481 L a competencia digital de los estudiantes universitarios Definicion conceptual y a nalisis de cinco instrumentos para su evaluacion/links/0912f50b33d967d777000 000.pdf

Ferro Soto, C., Martínez Senra, A. I. y Otero Neira, M. C. (2009). Ventajas del uso de las TICs en el proceso de enseñanza-aprendizaje desde la óptica de los docentes universitarios españoles. Edutec. Revista Electrónica de Tecnología Educativa, 0(29). Recuperado de http://www.edutec.es/revista/index.php/edutec-e/article/view/451

Freire, J. (2007). Los retos y oportunidades de la web 2.0 para las universidades. La Gran Guía de los Blogs 2008, 82-90. Recuperado de http://www.udc.gal/dep/bave/ifreire/pdf blog/Web\%202.0\%20y\%20universidad es\%20(JuanFreire GranGuiaBlogs).pdf

González Mariño, J. C. (2009). TIC y la transformación de la práctica educativa en el contexto de las sociedades del conocimiento. DIM: Didáctica, Innovación y Multimedia, (13). Recuperado dehttp://www.raco.cat/index.php/DIM/article/viewArticle/138942/0

González-Pérez, A. y De Pablos Pons, J. (2015). Factores que dificultan la integración de las TIC en las aulas. Revista de Investigación Educativa, 33(2), 401-417. https://doi.org/10.6018/rie.33.2.198161

Hall, R., Atkins, L. y Fraser, J. (2014). Defining a self-evaluation digital literacy framework for secondary educators: the DigiLit Leicester project. Research in Learning Technology, 22. Recuperado de http://www.researchinlearningtechnology.net/index.php/rlt/article/view/21440

Hernández Sampieri, R., Fernández Collado, C. y Baptista Lucio, P. (2010). Metodología de la investigación (5a ed). México, D.F: McGraw-Hill.

Kopcha, T. J. (2012). Teachers' perceptions of the barriers to technology integration and practices with technology under situated professional development. Computers \& 
Education, 59(4), 1109-1121. Recuperado de http://www.sciencedirect.com/science/article/pii/S0360131512001352

LOEI. (2011). Ley Orgánica de Educación Intercultural. Presidencia de la República del Ecuador. Ministerio de Educación y Ciencia. Educación Inclusiva. Recuperado de http://www.wipo.int/edocs/lexdocs/laws/es/ec/ec023es.pdf

LOES. (2010). Ley Orgánica de Educación Superior. Presidencia de la República del Ecuador. Recuperado de

http://www.ces.gob.ec/index.php?option=com phocadownload\&view=file\&id=651 \&Itemid $=564$

López Esteban, C. (2011). Mejores Prácticas en la Enseñanza de las Matemáticas: La integración de las TI Cs. [En línea]. Recuperado de http://scopeo.usal.es/enfoque-bol34-mejores-practicas-en-la-ensenanza-de-las-matematicas-la-integracion-de-las$\underline{\text { tics/ }}$

López Esteban, M. C. (2010). Las TICs y la comprensión matemática. El proceso de Bolonia y la educación comparada. Míradas críticas, 159, 74.

López García, J. C., \& Eduteka. (2003). La Integración de las TIC en Matemáticas. Recuperado de http://eduteka.icesi.edu.co/articulos/Editorial18

Mohammed Abdul, J. F. y Ramírez Velarded, R. V. (2009). Herramientas Web 2.0 para el Aprendizaje Colaborativo. Consultado el, 28. Recuperado de http://files.colegiocolombo-arabe8.webnode.com.co/200000034be12bbf0c9/herramientas\%20web\%202.0.pdf

Molina Martín, S. e Iglesias García, M. T. (2014). Una innovación didáctica en la universidad incorporando herramientas tecnológicas en Experiencias de Innovación Docente Universitaria. España: Ediciones Universidad de Salamanca. Recuperado de http://site.ebrary.com/lib/alltitles/docDetail.action?docID=10903617

Morón, J. L. (2013). Estrategias metodológicas para introducir las TIC y el Internet en matemáticas. Recuperado de http://repositorioacademico.upc.edu.pe/upc/handle/10757/285380

Pachler, N., Cook, J. y Bachmair, B. (2010). Appropriation of Mobile Cultural Resources for Learning: International Journal of Mobile and Blended Learning, 2(1), 1-21. https://doi.org/10.4018/imbl.2010010101

Padilla Partida, S., Moreno, C. I. y Hernández Castañeda, R. (2015). Barreras para la integración de buenas prácticas con TIC. Estudio de caso. Innoeduca. International Journal of Technology and Educational Innovation, 1(2), 80-90. Recuperado de https://dialnet.unirioja.es/descarga/articulo/5363138.pdf

Pérez Escoda, A., Castro Zubizarreta, A. y Fandos Igado, M. (2016). La competencia digital de la Generación Z: claves para su introducción curricular en la Educación Primaria.

Prensky, M. (2001). Digital natives, digital immigrants. On the Horizon, 9(5), 1-6.

Revelo Rosero, J. E. (2017). Modelo de integración de la competencia digital docente en la enseñanza de la matemática en la universidad tecnologica equinoccial (Doctoral dissertation). Universidad de Extremadura. Recuperado de 
http://dehesa.unex.es/bitstream/handle/10662/6214/TDUEX 2017 Revelo Roser o.pdf? sequence $=1$

Revelo Rosero, J. E., Revuelta Domínguez, F. I. y González-Pérez, A. (2018). Modelo de integración de la competencia digital del docente universitario para su desarrollo profesional en la enseñanza de la matemática - Universidad Tecnológica Equinoccial de Ecuador. EDMETIC, 7(1), 196-224. https://doi.org/10.21071/edmetic.v7i1.6910

Revuelta Domínguez, F. I. y Pérez Sánchez, L. (2009). Interactividad en los entornos de formación on-line. España: Editorial UOC. Recuperado de http://site.ebrary.com/lib/alltitles/docDetail.action?docID=10646727

Rodríguez Izquierdo, R. M. (2010). El impacto de las TIC en la transformación de la enseñanza universitaria: repensar los modelos de enseñanza y aprendizaje. Teoría de la Educación. Educación y Cultura en la Sociedad de la Información, 11(1).

Rodríguez Pérez, I. (2015). La incorporación de la web 2.0 en la práctica educativa. Revista Iberoamericana para la Investigación y el Desarrollo Educativo ISSN: 2007-2619, (11). Recuperado de http://ride.org.mx/111/index.php/RIDESECUNDARIO/article/download/691/676

Romero Fernández, L. M. (2008). Gestión de Conocimientos Universitarios y web 2.0 en el núcleo de la prospectiva de la educación a distancia. Memorias II Congreso CREAD ANDES y II Encuentro Virtual Educa Ecuador. Recuperado de http://reposital.cuaed.unam.mx:8080/jspui/handle/123456789/2785

Romeu Fontanillas, T. (2011). La Docencia en colaboración en contextos virtuales: estudio de caso de un equipo de docentes del área de competencias digitales de la UOC (Doctoral dissertation). Universitat Oberta de Catalunya. Recuperado de https://openaccess.uoc.edu/webapps/o2/handle/10609/18101

Salinas, J. (2004). Cambios metodológicos con las TIC. Estrategias didácticas y entornos virtuales de enseñanza-aprendizaje. Bordón, 56(3-4), 469-481. Recuperado de http://www.researchgate.net/profile/Jesus Salinas/publication/39214325 Cambio s metodolgicos con las TIC estrategias didcticas y entornos virtuales de enseanz a-aprendizaje/links/0912f509c0a81c366d000000.pdf

Salinas, J., Benito, B. de y Lizana, A. (2014). Competencias docentes para los nuevos escenarios de aprendizaje. Recuperado 23 de noviembre de 2015, de http://148.215.2.11/articulo.oa?id=27431190010

Sangeeta Namdev, D. (2012). ICT and Web Technology Based Innovations in Education Sector. Turkish online journal of distance education, 13(4), 256-268. Recuperado de http://eric.ed.gov/?id=EJ1000427

Santamaría González, F. (2005). Herramientas colaborativas para la enseñanza usando tecnologías web: weblogs, redes sociales, wikis, web 2.0. Recuperado de http://cursa.ihmc.us/rid=1196863010187 1551044424 8326/Herramientas Web 2-0.pdf

SCOPEO. (2012). e-MatemáTICas. Salamanca: Scopeo Monográfico No. 4. Recuperado de http://scopeo.usal.es/wp-content/uploads/2013/04/scopeom004.pdf 
Silva Quiroz, J., Miranda, P., Gisbert, M., Morales, J. y Onetto, A. (2016). Indicadores para evaluar la competencia digital docente en la formación inicial en el contexto Chileno - Uruguayo / Indicators to Assess Digital Competence of Teachers in Initial Training in the Chile - Uruguay Contex. Revista Latinoamericana de Tecnología Educativa RELATEC, 15(3), 55-67. https://doi.org/10.17398/1695-288X.15.3.55

Sosa Díaz, M. J. (2015). El proceso de integración de las tecnologías de la información y comunicación en centros de Educación Primaria: Estudio de caso múltiple (Tesis Doctorado). Departamento de Ciencias de la Educación. Universidad de Extremadura, Cáceres. Recuperado de http://dialnet.unirioja.es/servlet/tesis?codigo=45464

Tapscott, D. (2010). Grown Up Digital. How the Net Generation Is Changing Your World. International Journal of Market Research, 52(1), 139.

Tello Leal, E., Reyna, S., M, C., Lucio Castillo, M., Morelos, F., \& Magdalena, M. (2010). Análisis de los servicios de la tecnología Web 2.0 aplicados a la educación. No Solo Usabilidad, (9). Recuperado de http://www.nosolousabilidad.com/articulos/tecnologia educacion.htm

UNESCO. (2008). Estándares UNESCO de competencia en TIC para docentes. Londres: Organización de las Naciones Unidas para la Educación, la Ciencia y la Cultura. Recuperado de http://eduteka.icesi.edu.co/pdfdir/UNESCOEstandaresDocentes.pdf

UNESCO. (2011). Alfabetización mediática e informacional: curriculum para profesores. Paris: Organización de las Naciones Unidas para la Educación, la Ciencia y la Cultura, UNESCO.

Wodzicki, K., Schwämmlein, E. y Moskaliuk, J. (2012). “Actually, I Wanted to Learn”: Studyrelated knowledge exchange on social networking sites. Social Media in Higher Education, 15(1), 9-14. https://doi.org/10.1016/j.iheduc.2011.05.008

Wong, E. M., Li, S. S., Choi, T. y Lee, T. (2008). Insights into Innovative Classroom Practices with ICT: Identifying the Impetus for Change. Educational Technology \& Society, 11(1), 248-265. Recuperado de http://www.jstor.org/stable/jeductechsoci.11.1.248

Yang, H. (2012). ICT in English schools: transforming education? 1. Technology, pedagogy and education, 21(1), 101-118. Recuperado de http://www.tandfonline.com/doi/abs/10.1080/1475939X.2012.659886

Zuluaga, J. M., Pérez, F. E. y\& Gómez, J. D. (2012). Uso de la web 2.0 para la construcción de blogs, páginas web y animaciones. Recuperado de http://files.artematic3.webnode.es/200000013-4c4974d42b/Artematic-Taller\%201\%20uso\%20de\%20la\%20web\%202.0\%20U\%20de\%20M\%202014.pdf 


\section{Autores}

JORGE REVELO-ROSERO obtuvo el título de Doctor (PhD) en Formación del Profesorado y TIC en Educación por la Facultad de Formación del Profesorado de la Universidad de Extremadura (España) en 2017. Obtuvo su título de Magíster en Contabilidad y Auditoría CPA en la Universidad UTE (Ecuador) en 2003. Obtuvo el título de Licenciado en Ciencias de la Educación, Profesor de Enseñanza Media en la Especialización de Matemáticas y Física por la Facultad de Filosofía, Letras y Ciencias de la Educación de la Universidad Central del Ecuador en 1993.

Actualmente es profesor titular de la Universidad UTE de Quito. Sus principales temas de investigación incluyen las áreas de matemáticas y afines, aplicaciones matemáticas para ingeniería y física, investigación de modelos educativos con TIC. Es autor de varios de capítulos de libros y artículos publicados en revistas de alto impacto (Emerging Source Citation Index, Latindex, Redalcy, Scielo, Scopus).

SONIA CARRILlo PUGA obtuvo el título de Licenciada en Ciencias de la Educación, especialización Educación Inicial y Parvularia en la Universidad Técnica de Machala (Ecuador). Obtuvo el título de Magister en Gerencia Educativa en la Universidad Técnica de Machala (Ecuador).

Actualmente es profesora contratada de la Unidad Académica de Ciencias Sociales de la Universidad Técnica de Machala (Ecuador). Sus principales temas de investigación incluyen el desarrollo de estrategia metacognitivas para mejorar el nivel de comprensión lectora y hermenéutica. Es autora de varios de capítulos de libros y artículos publicados en revistas de alto impacto (Emerging Source Citation Index, Latindex). 